\title{
Modification of Saccades Evoked by Stimulation of Frontal Eye Field during Invisible Target Tracking
}

\author{
Andrei Barborica and Vincent P. Ferrera \\ Department of Psychiatry, Center for Neurobiology and Behavior, Keck-Mahoney Center for Brain and Behavior Research, Columbia University, New York, \\ New York 10032
}

\begin{abstract}
We investigated the internal representation of invisible moving targets using electrical microstimulation in the prefrontal cortex. Monkeys were trained to make saccades to the extrapolated position of a small moving target that was rendered invisible during part of its trajectory. Although the target was invisible, involuntary saccades were evoked by electrical microstimulation of the frontal eye field. Stimulation was applied at different times relative to the disappearance of the target while the monkey fixated. When stimulation was applied immediately after target disappearance, electrically evoked saccades were biased toward the starting point of the target trajectory. When stimulation was applied later in the trial, evoked saccades were biased toward the end of the trajectory. The bias in evoked saccade direction changed continuously over time. The magnitude and statistical significance of the electrically evoked saccade deviation depended on the accuracy of the monkeys' voluntary saccades relative to the invisible target. The results suggest that covert tracking is accompanied by a continuously shifting saccade plan that moves along the target path.
\end{abstract}

Key words: monkey; frontal eye field; stimulation; prediction; saccade; planning

\section{Introduction}

The ability to predict the future location of moving targets is important for visually guided behavior. Humans and monkeys are able to predict target motion accurately even when the target is rendered temporarily invisible (Filion et al., 1996). Schizophrenia patients show an impaired ability to estimate invisible target motion (Hooker and Park, 2000). This ability may depend on an internal representation of the invisible target or of the motor plan required to intercept the target. Electrical stimulation of oculomotor centers may allow one to probe such intermediate representations. Stimulation in the intermediate layers of the superior colliculus or frontal eye field (FEF) tends to produce fixed vector saccades in awake but passive monkeys (Robinson and Fuchs, 1969; Robinson, 1972; Bruce et al., 1985). However, electrically evoked saccades may be modified when monkeys perform a task that requires a voluntary eye movement response (Kustov and Robinson, 1996; Gold and Shadlen, 2000, 2003). In these cases, the evoked saccade is a weighted average of the fixed vector and voluntary saccades. Modification of electrically evoked saccades can, therefore, be a useful method for revealing covert saccade plans.

In the current study, monkeys were trained to make saccades to the extrapolated location of a small moving target that was rendered invisible during part of its trajectory. This motion extrapolation task required that a voluntary saccade be initiated

Received July 30, 2003; revised Feb. 22, 2004; accepted Feb. 22, 2004.

This work was supported by the Whitehall Foundation and National Institutes of Health Grant MH59244. We thank $\mathrm{G}$. Case for designing and fabricating the multi-electrodes used in this study. J. Willi and A. Banta provided excellent technical assistance.

Correspondence should be addressed to Dr. Vincent P. Ferrera, Center for Neurobiology and Behavior, Columbia University, Kolb Annex 565, 1051 Riverside Drive, New York, NY 10032. E-mail: vpf3@columbia.edu.

D0I:10.1523/JNEUROSCI.4702-03.2004

Copyright $\odot 2004$ Society for Neuroscience $\quad 0270-6474 / 04 / 243260-08 \$ 15.00 / 0$ while the target was still invisible (Barborica and Ferrera, 2003). The amount of time that the target was invisible varied randomly. In initial training, the direction and speed of the moving target also varied so that monkeys learned to adjust saccade amplitude and direction according to target direction, speed, and duration of invisibility. The predictive saccade was always directed toward a location away from the initial position of the target (i.e., for horizontal target motion, the saccade would be directed toward the visual hemifield opposite to that in which the target initially appeared). One might, therefore, expect that the direction of the planned saccade would be toward the end point of the target trajectory and would remain constant for the duration of the trial.

While the target was invisible, involuntary saccades were evoked by electrical microstimulation of the FEF. The target trajectory was chosen to be as close as possible to perpendicular relative to the direction of the fixed vector saccade (Fig. 1A). Stimulation was applied at different times relative to the disappearance of the target. When stimulation was applied immediately after target disappearance, evoked saccades were biased toward the starting point of the target trajectory. When stimulation was applied later, saccades were biased toward the end of the trajectory. The bias in evoked saccade direction changed continuously over time and was nearly symmetrical for opposite directions of target motion. The results suggest that when monkeys are planning saccades to invisible moving targets, the direction of the planned saccade changes over time as if the locus of attention or motor planning were moving continuously along the target path.

These experiments have been reported in abstract form (Barborica and Ferrera, 2002).

\section{Materials and Methods}

Experiments were performed on three juvenile male rhesus monkeys (Macaca mulatta). All methods were approved by the Institutional Ani- 


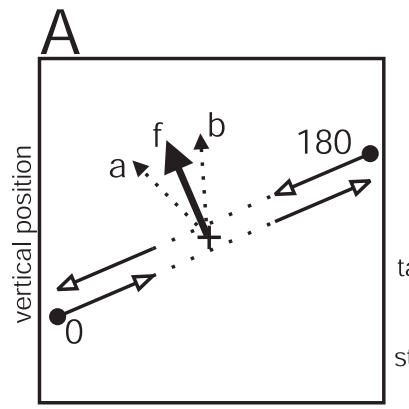

B

horizontal position

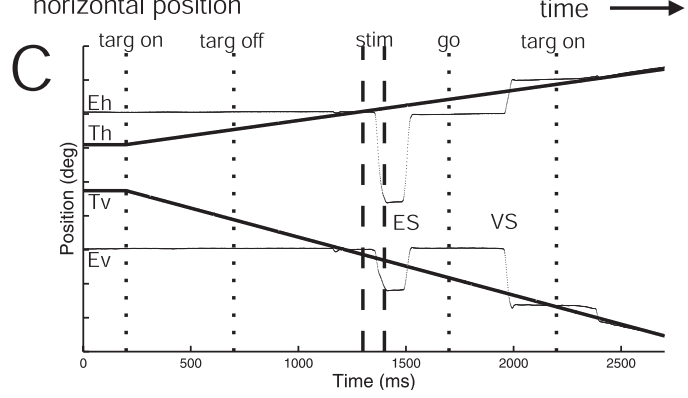

Figure 1. Motion extrapolation task and stimulation protocol. $A$, Depiction of video display showing target trajectories (open arrows). The target paths for the two directions of motion are shown with a slight separation for clarity. In the actual experiment, the paths overlapped. The solid lines indicate portions of the trajectory in which the target was visible. The dotted lines indicate where the target was invisible. The arrow labeled $f$ represents a hypothetical fixed vector saccade evoked by stimulating FEF when the monkey was fixating a stationary target. The arrows labeled $a$ and $b$ indicate hypothetical saccades that might be evoked by microstimulation when the monkey was performing the motion extrapolation task. The large + indicates the fixation mark. $B$, Trial time structure. Trials were divided into cue, delay, stim/re-fix, and saccade epochs. The traces indicate the state (on/off) of the fixation spot (fix), moving target (targ), microstimulation (stim). C, Eye and target position traces for a single stimulation trial. Eh and Th are horizontal eye and target position, and Ev and Tv are vertical eye and target position. The traces are offset vertically for clarity. The $y$-axis tick marks represent $5^{\circ}$ increments. ES and VS are the electrically evoked and voluntary saccades, respectively. The vertical dotted and

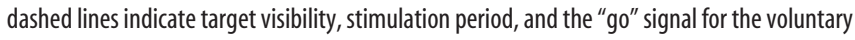
saccade.

mal Care and Use Committee at Columbia University and the New York State Psychiatric Institute. Monkeys were prepared for experiments by surgical implantation of a post used for head restraint and a recording chamber to give access to the cortex. Recording chambers were placed at stereotaxic coordinates 20 anterior, 15 lateral (Robinson and Fuchs, 1969). The position of the arcuate sulcus within the recording chamber was visualized through a craniotomy made during the surgery. Eye position was recorded using a monocular scleral search coil (Robinson, 1963; Judge et al. 1980). Monkeys were trained to sit in a primate chair for the duration of the experiment with their heads restrained and to perform visual fixation and tracking tasks for liquid reward.

Visual stimulation. Visual stimuli were generated and controlled by a Cambridge Research Systems (Cambridge, UK) VSG2/3F video frame buffer. The output from the video board was displayed on a calibrated color monitor with a $60 \mathrm{~Hz}$ non-interlaced refresh rate. The spatial resolution of the display was 1280 pixels by 1024 lines. The central fixation target was a $0.5^{\circ}$ white square (luminance, $65 \mathrm{~cd} / \mathrm{m}^{2}$ ) presented on a uniform dark background. Moving targets were $1.0^{\circ}$ yellow squares (luminance, $15 \mathrm{~cd} / \mathrm{m}^{2}$ ). To obtain the smoothest target motion onscreen, the target was allowed to move a fixed integer number of pixels/lines on every display frame. As a result, the desired target speeds were coerced to the nearest speed that resulted in a smooth target motion. The coerced speed depended on the direction of motion, therefore across all experiments there was a distribution of actual target speeds that for a "desired" target speed of $10 \% \mathrm{sec}$ could extend from $9.6 \% \mathrm{sec}$ to $12.2 \% \mathrm{sec}$. The frame buffer was programmed to send out digital pulses (frame sync) for timing purposes at the beginning of each video frame in which a target was turned on or off. These pulses were recorded by the computer using a hardware timer and stored together with the eye movement data.

Behavioral task. Monkeys were trained to perform a task in which they were shown a small moving target on a video display (Fig. $1 \mathrm{~A}$ ). The target moved for $500 \mathrm{msec}$, disappeared for 1250-1750 msec (uniform random distribution), and then reappeared moving along the same linear trajectory, as if it had passed behind an occluder (Fig. $1 B$ ). The monkeys' task was to fixate a stationary spot in the center of the display during the initial target motion and the first 750-1250 msec of the occlusion interval. At a random time, the fixation spot disappeared, and this served as the "go" signal for the monkey to initiate a saccade to the extrapolated position of the invisible moving target. There was a grace period of $500 \mathrm{msec}$ after the "go" signal during which there was no fixation requirement. The grace period ended with the reappearance of the moving target. After the grace period, eye position was required to be within a square fixation window of \pm 4.0 or $\pm 5.0^{\circ}$ centered on the target location. When the moving target reappeared, it was at least $7.5^{\circ}$ from the location of the fixation spot. After the "go" signal, the monkey was allowed to make any combination of saccades and smooth eye movements to acquire the target.

During training, target direction and speed, as well as the occlusion interval duration, were selected randomly in each trial. Target direction was chosen from a set of eight directions separated by $45^{\circ}$. Target speed was chosen from a set of four speeds close to $5,10,15$, and $20 \% \mathrm{sec}$. Occlusion duration was an integral number of video frames selected randomly between 1250 and $1750 \mathrm{msec}$. This training was undertaken to reduce the likelihood of the monkeys performing saccades to a fixed location. The monkey was most likely to be rewarded by making a saccade appropriate for the target direction, speed, and occlusion duration selected for each trial. Analysis of saccade direction and amplitude confirmed that this was the case.

During stimulation experiments, only a single speed and axis (two opposing directions) of target motion were used for any given stimulation site. The axis of motion was chosen to be perpendicular to the direction of the fixed vector saccade evoked by stimulation during a simple fixation task. Occlusion interval duration varied randomly, as in training.

Monkeys were also tested on a standard memory-guided saccade task with stationary targets. The temporal structure of this task was identical to the motion extrapolation task, with a memory interval (corresponding to the occlusion interval) of variable duration (1250-1750 msec). On each trial, the target location was selected from an array of eight positions separated by $45^{\circ}$ around the clockface. Target eccentricity was $10^{\circ}$. Visual feedback was provided by re-illuminating the saccade target after the memory-guided saccade was executed. Neural activity was recorded during the memory-guided saccade task in the absence of electrical stimulation to determine the spatial tuning of neurons at the stimulation site. In separate experiments, electrical stimulation was applied during memoryguided saccades. For these stimulation experiments, only two opposing target locations were used.

Electrical microstimulation. Parylene-coated tungsten single electrodes or epoxy-coated tungsten multi-electrodes with impedances typically 0.5 $\mathrm{M} \Omega$ were used in microstimulation experiments. Stimulation experiments were performed during the course of neuronal recording studies. Sites in FEF were located in gray matter on the anterior bank of the arcuate sulcus and were stimulated through the same electrode used to record neuronal activity. The stimulation consisted of a $67 \mathrm{msec}$ (four video frames) train of biphasic pulses $(0.2 \mathrm{msec}$ per phase; cathode leading) at a rate of 350 pulses/sec (24 pulses total) and was delivered by an optically isolated pulse stimulator with output compliance up to $100 \mathrm{~V}$ (A-M Systems, Everett, WA). The stimulator was gated by a computergenerated digital signal so as to be synchronized with other trial events. The threshold for evoking saccades at each FEF site was determined during a simple fixation task. The amplitude of the stimulation current at each site was adjusted to be near or just above the threshold for evoking saccades (pulse amplitude range, 10-100 microamps; one-half peak to peak).

For stimulation during the motion extrapolation task, the fixation requirement was turned off at the onset of the electrical stimulus and remained off until the reappearance of the moving target (i.e., $500 \mathrm{msec}$ 
after the "go" signal). The central fixation spot remained illuminated during stimulation. Stimulation was applied after a delay of 0,100 , $200,300,400,500,600$, or $700 \mathrm{msec}$ relative to the start of the occlusion interval (for brevity, this delay is also referred to as "gap duration"). Stimulation trials were interleaved randomly with nonstimulated trials, which had exactly the same structure except (1) the electrical stimulus was absent, (2) the fixation requirement remained in effect until the "go" signal, and (3) the size of the fixation window when the moving target reappeared was $\pm 5^{\circ}$ for stimulation trials versus $\pm 4^{\circ}$ for nonstimulation trials (the smaller window helped reinforce correct behavior). A block of trials consisted of 7 stimulation trials (one of each delay duration) plus 1 nonstimulated trial for each of two opposite directions of motion, for a total of 16 trials. Generally, data were collected for 10-12 blocks of trials at each stimulation site. Eye position traces for a typical stimulation trial are shown in Figure $1 C$.

Eye movement recording and analysis. Eye position was monitored using a monocular scleral search coil system (CNC Engineering). Separate horizontal and vertical eye position signals were fed through an analog differentiator (lowpass, $-3 \mathrm{~dB}$ at $25 \mathrm{~Hz}$ ) to yield horizontal and vertical eye velocity. The eye position and eye velocity signals were then digitally sampled by computer at $1 \mathrm{kHz} /$ channel and stored on disk for offline analysis. Eye position and velocity records were used to estimate saccade parameters. Polar eye velocity $\left(\mathrm{R}^{\prime \prime}\right)$ was constructed by combining the magnitudes of the horizontal $\left(\mathrm{H}^{\prime}\right)$ and vertical $\left(\mathrm{V}^{\prime}\right)$ eye velocity. Polar eye velocity was digitally differentiated to yield polar eye acceleration $\left(\mathrm{R}^{\prime \prime}\right)$. For other saccades, movement onset was computed using an acceleration criterion $\left(\mathrm{R}^{\prime \prime} \geq 500^{\circ} / \mathrm{sec}^{2}\right)$. Saccade offset was found using the complementary criterion $\left(\mathrm{R}^{\prime \prime} \leq 500^{\circ} / \mathrm{sec}^{2}\right)$. This criterion was not always appropriate for small saccades; hence, each trial was also checked by visual inspection. Initial and final eye positions were obtained by taking the average eye position in a $10 \mathrm{msec}$ window before the beginning or after the end of the saccade, respectively. Electrically evoked saccades were included in the analysis only if they were $>1.5^{\circ}$ in amplitude.

Regression analysis. Regression lines were fit using a method based on principal components analysis (PCA). This method minimizes the error in both the $x$ and $y$ variables as opposed simple regression, which minimizes the error in the ordinate only and is known to produce systematic biases in the estimates of slope and intercept (Snedecor and Cochran, 1989). Simple regression estimates the most likely saccade end point for a given target eccentricity, whereas PCA regression may be better suited for the inverse problem of estimating target position based on the saccade end points.

\section{Results}

Behavioral analysis of voluntary saccades initiated during the occlusion interval

To gain insight into the strategy used to perform the task, we analyzed voluntary saccades to invisible moving targets in the absence of stimulation. Behavioral data were collected in 106 sessions (75 for monkey A, 17 for monkey C, 14 for monkey D). For these sessions, the target direction varied among eight possi-

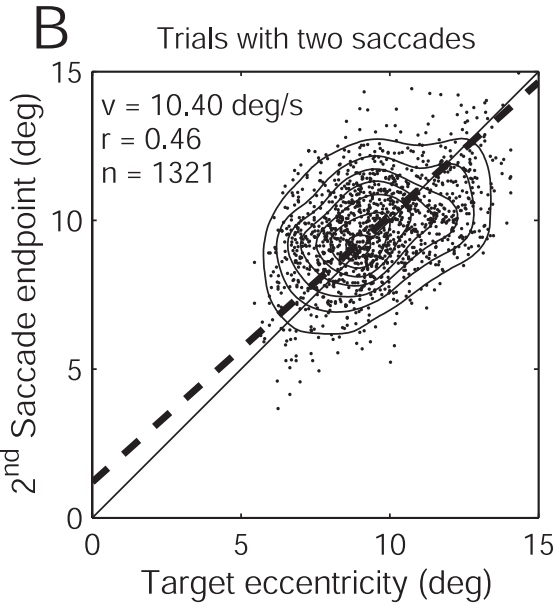

D

All trials

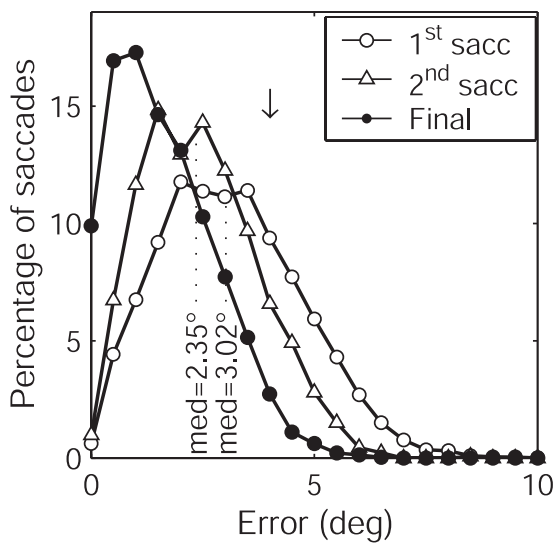

Figure 2. Behavioral analysis of saccades to invisible moving targets. $A$, Eye position eccentricity after the first saccade versus

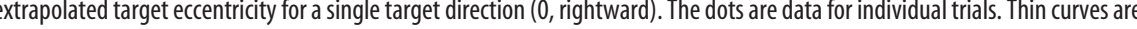
iso-density contours. The legend shows the correlation coefficient $(r)$, target speed (v), and number of saccades ( $n$ ). The dashed ( position regarding target position) after the first and second saccades for trials with at least two saccades. The legend indicates the number of trials $(\mathrm{n})$. The thin line is $x=y$. D, Distribution of errors (eye position relative to invisible target position) after the first saccade (open circles), second saccade (open triangles), or just before the reappearance of the target (Final). The dotted lines indicate the median error for the first and second saccades. The downward arrow indicates the size of the fixation window $\left(4^{\circ}\right)$.

ble directions, and the occlusion interval varied from 1250 to $1750 \mathrm{msec}$ (uniformly distributed). The eye movement response to invisible targets could include multiple saccades as well as smooth eye movements. The first voluntary saccade averaged $9.2^{\circ}$ $( \pm 1.8 \mathrm{SD} ; n=6438)$ in amplitude and had a median error of $3.02^{\circ}$ relative to the extrapolated invisible target position.

The eye position eccentricity after the first saccade ("saccade end point," for short) was significantly correlated with invisible target eccentricity $(r=0.34 ; n=6438 ; p<0.0001)$. Figure $2 \mathrm{~A}$ shows data for all three monkeys with the target moving horizontally to the right. The correlation $(r=0.54)$ is higher than for the entire data set because we found that there was a directional anisotropy such that performance was better for horizontally moving targets (both directions) than for targets moving in other directions. The data were fit with PCA regression lines (Fig. $2 \mathrm{~A}$, dashed line). For the data in Figure $2 A$, the regression slope was 1.1. For the entire data set (all target directions combined), the slope was 1.0.

On 1321 of 6438 (20.5\%) trials, a second saccade was initiated before the end of the occlusion interval (Fig. $2 B$ ). The eye posi- 

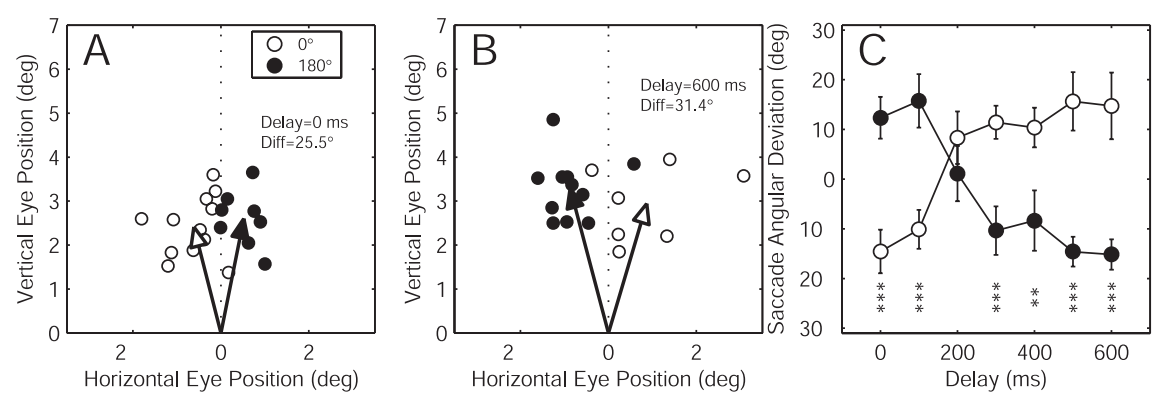

Figure 3. Effects of microstimulation during invisible target tracking at a single FEF site. $A$, Stimulation was applied at the beginning of the occlusion interval. Each data point is a single saccade end point. The open and closed symbols correspond to the two directions of target motion. The arrows indicate the center of mass of the respective data points. B, Stimulation 600 msec after the beginning of occlusion interval. $C$, Average saccade deviation at different times during the occlusion interval. The error bars are \pm 1 SEM. The asterisks indicate the $t$ test significance level $\left({ }^{*} p<0.05 ;{ }^{* *} p<0.01{ }^{* * *} p<0.001\right)$.

tion eccentricity after the second saccade averaged $9.7^{\circ} \pm 1.6 \mathrm{SD}$ and tended to be significantly larger than the eccentricity after the first saccade in the same trial (mean, $9.2^{\circ} \pm 1.9 \mathrm{SD}$; paired $t$ test, $\left.p \ll 10^{-5}\right)$. The eye position eccentricity after the second saccade was more strongly correlated with target eccentricity $(r=0.46$; $n=1321 ; p<0.0001)$ than was eye position after the first saccade (Fig. $2 B$ ). The median error after the second saccade was $2.35^{\circ}$ (Fig. 2C). Second saccades tended to occur in trials in which the first saccade error was significantly larger than the overall average (mean error, 3.7 vs $3.1^{\circ}$; unpaired $t$ test, $p \ll 10^{-5}$ ). For trials with two saccades, the second saccade reduced the error (eye position regarding target position) by an average of $1.3^{\circ}$ (Fig. $2 C$ ), and this reduction was highly significant (paired $t$ test, $p \ll$ $\left.10^{-5}\right)$.

If monkeys had used a strategy of making saccades to a fixed location, one might expect that the first saccade end points would provide an initial estimate of that location and that the second saccade end points would be even closer to that fixed location. Under this strategy, the average eye position eccentricity after the second saccade should be the same as it was after the first saccade, which was not the case (see above). Furthermore, the fixed target hypothesis predicts that the second saccade should reduce the error relative to the target position after the first saccade. In fact, relative to the target position after the first saccade, the first and second saccades both tended to have the same error (paired $t$ test, $p=0.45$ ). In other words, the second saccade did not bring the eye closer to the target location as estimated by the first saccade. Rather, the second saccade brought the eye closer to where the target was heading. These observations suggest that the error signal that drove the second saccade took into account the future position of the target.

An essential feature of the behavioral paradigm was that the monkeys had a relatively long grace period ( $500 \mathrm{msec})$ between the time of the "go" signal and the time when the fixation window was imposed. During this period, the monkey could look anywhere. It is important to show that behavior was not artificially constrained by the fixation window. This constraint might lead to unintended screening of the data. If the monkey made random eye movements and only those that happened to land within a moving window were selected, then a correlation would arise perforce. Error trials cannot be used to address this issue because the monkey may have failed to attend to the task altogether. Instead, we looked at the eye position error just before the fixation window was re-imposed at the end of the occlusion interval. The distribution of these errors is shown in Figure $2 D$ (curve labeled "Final"), along with the error distributions for the first and second saccades. The downward arrow indicates the size of the fixation window $\left( \pm 4^{\circ}\right)$. The median error $\left(1.45^{\circ} ; n=\right.$ 6481) was far smaller than the size of the fixation window, and the distribution showed no evidence of truncation, as one would expect if the data had been clipped by the window. (The tail of the distribution arises because of the square shape of the window, which allows for saccades falling into the corners to have errors up to 1.41 times the nominal window size.) Additional sessions were run with fixation window sizes of \pm 3 or $\pm 5^{\circ}$. The median error varied only slightly $\left(1.3^{\circ}\right.$ for a $\pm 3^{\circ}$ window vs $1.6^{\circ}$ for a $\pm 5^{\circ}$ window). One would expect that a larger fixation window might result in less accurate saccades. However, the correlation between eye and target position after the first saccade was slightly better for the $5^{\circ}$ window $(r=0.36)$ than for the $4^{\circ}$ window $(r=$ $0.34)$. Overall, the data suggest that the task requirements set fairly liberal constraints on performance, within which monkeys behaved as they might have under natural conditions.

\section{Deviation of electrically evoked saccades}

To map the FEF, we performed a total of 244 stimulation experiments in three monkeys ( 83 in monkey A, 77 in monkey C, 84 in monkey D) at 156 distinct cortical sites (46 in monkey A, 52 in monkey C, 58 in monkey D). All 156 sites were tested by stimulating during a simple fixation task (Opris et al., 2001) to determine the threshold for evoking saccades. Saccades were evoked at 114 of 156 (73\%) sites (46 in monkey A, 32 in monkey C, 36 in monkey D), of which 56 (27 in monkey A, 16 in monkey C,13 in monkey D) had thresholds between 10 and $50 \mu \mathrm{A}$, whereas 58 (19 in monkey A, 16 in monkey C, 23 in monkey D) had thresholds between 50 and $100 \mu \mathrm{A}$. The average stimulation thresholds were 40,60 , and $70 \mu \mathrm{A}$ for monkeys $\mathrm{A}, \mathrm{C}$, and $\mathrm{D}$, respectively. These results indicate that most stimulation sites were in or near the functionally defined FEF (Bruce et al., 1985; Stanton et al., 1989).

In the invisible moving target experiment, the target trajectories were oriented orthogonal to the direction of the electrically evoked saccade. The total number of stimulation sites was 51 [25 in monkey C, 24 in monkey D; only 2 sites were tested in a third monkey (A), but the results were consistent with the other two monkeys]. There were 10,847 stimulation trials, and saccades (minimum amplitude, $1.5^{\circ}$ ) were evoked during the stimulation period in 6396 trials (59\%). This confirms that, on average, the stimulation current was just above the motor threshold [defined as the current level needed to evoke saccades in $50 \%$ of the trials (Bruce et al. 1985)].

Figure 3 shows the results of stimulating a single FEF site during the imaginary target task. The characteristic saccade vector for this site pointed directly upward. The angular deviation was measured as the direction of the evoked saccade during imaginary target tracking relative to the mean direction of the saccades evoked during a simple fixation task (Opris et al., 2001). Previous studies have measured changes in saccade end point (Kustov and Robinson, 1996; Gold and Shadlen, 2000, 2003), whereas the current study used saccade direction. The magnitude of the shift in saccade end points may vary with saccade amplitude with the result that large amplitude saccades dominate the population average. The use of saccade direction facilitates comparison of results across sites with different characteristic saccade amplitudes. 

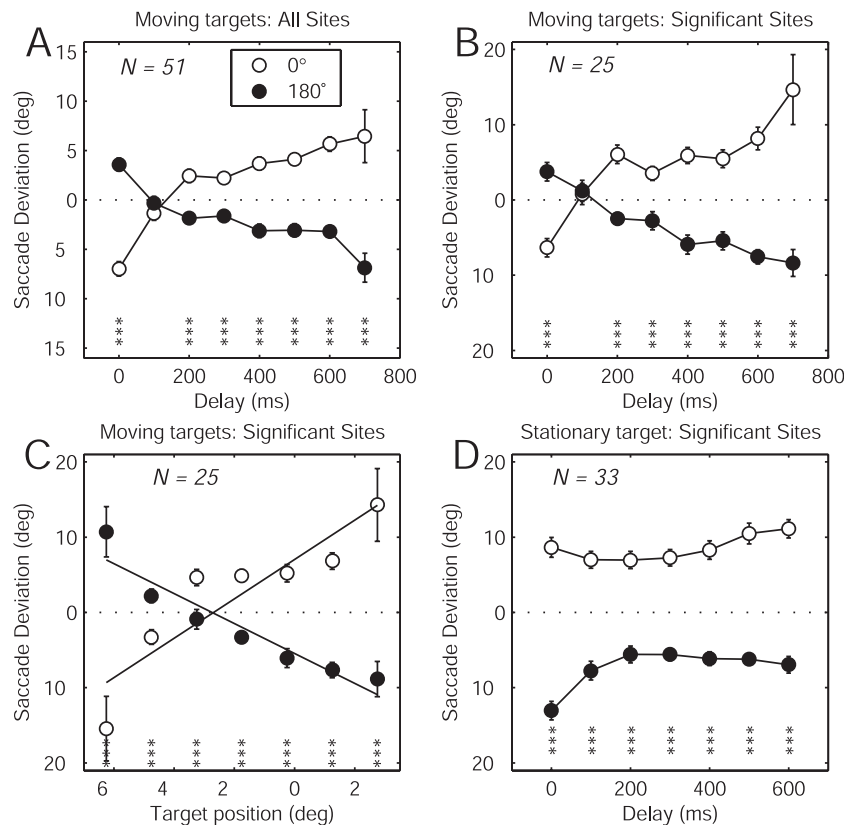

Figure 4. Directional bias of evoked saccade as a function of stimulation onset time. A, All sites tested with orthogonal target motion. $B$, Sites with a significant $(p<0.05)$ effect of target direction. The error bars are \pm 1 SEM. The asterisks indicate the $t$ test significance level $\left({ }^{*} p<\right.$ $\left.0.05 ;{ }^{* *} p<0.01 ;{ }^{* * *} p<0.001\right)$. C, Relationship between evoked saccade deviation and invisible target position. The lines indicate best-fitting simple linear regressions. D, Stimulation during the delay interval of a memory-guided saccade task. The conventions are the same as in $A$ and $B$. Note that the $y$-axis scale in $A$ is different from the other panels.

When stimulation was applied at different time points during the occlusion interval ("delay" and "gap duration" both refer to the time between the start of the occlusion interval and the onset of microstimulation), there were two effects: (1) the direction of the saccade bias reversed itself over time (Fig. 3, compare $A, B$ ); and (2) the angle of the bias changed over time (Fig. 3C). These effects can be summarized simply by noting that the evoked saccade bias was correlated with the position of the imaginary target at the time of stimulation.

For each site, a two-way ANOVA was used to test the effects of target direction and delay duration on evoked saccade deviation. Target direction had a significant effect $(p<0.05)$ at 25 of 51 $(49 \%)$ sites, whereas delay duration had a significant effect at 18 of $51(35 \%)$ sites.

Results for all 51 stimulation sites were combined by averaging the saccade deviations for each direction of target motion and gap duration (Fig. 4A). To combine saccade data across sites, saccade deviations were rotated through an angle equal and opposite to the direction of the characteristic saccade vector. Figure $4 B$ shows the average deviations for sites that had a statistically significant effect of target direction in the two-way ANOVA (see above). These sites were not selected on the basis of the significance of the gap effect. Nevertheless, there is a quasi-linear trend for saccade deviation versus gap duration.

How well was evoked saccade deviation correlated with the position of the invisible target? This question is partly answered by the relationship between evoked saccade deviation and gap duration, because there was a direct correspondence between gap duration and target position for any given experiment. However, because of variability in the target speed and initial position between experiments (see Materials and Methods), the relationship between gap duration and target position at the population level was not perfect. A given gap duration could include target posi- tions that varied by up to $2^{\circ}$ of visual angle. We, therefore, performed a direct comparison of saccade deviation and invisible target position. We grouped the invisible target positions into $1.5^{\circ}$ bins and averaged the evoked saccade deviation within each bin. The results are shown in Figure $4 C$ for sites with a significant effect of target direction. The large error bars for the data points at the ends of the invisible target position range are attributable to the expectedly fewer data points in those bins. The correlation between evoked saccade deviation and invisible target position was strong $\left(r=0.90\right.$ and -0.95 for 0 and $180^{\circ}$ target directions, respectively). The intersection of the regression lines occurred at $-2.5^{\circ}$, indicating that the planned saccade tended to lead the invisible target. For these same trials, the voluntary saccade overshot the invisible target by an average of $1.39^{\circ}$.

In the motion extrapolation task, target position covaried with elapsed time. It is difficult to determine whether the electrical saccade deviation varied with target position or simply changed as a function of time during the occlusion interval. We, therefore, did an experiment in which target position was fixed and only the time of stimulation was varied. We applied stimulation during the delay interval of a memory-saccade task with stationary targets. In this task, there was no target motion, and the cue and target locations were identical. The time structure of the task was identical to the motion task. Target positions were chosen so that the memory-guided saccades would be approximately perpendicular to the electrically evoked saccades. A total of 36 sites were tested in two monkeys (C and D). There were a total of 5589 trials, of which 3223 (58\%) contained electrically evoked saccades. Hence, on average, the current level relative to motor threshold was similar to the first experiment. Of the 36 sites tested, $33(92 \%)$ showed a significant effect of target direction, whereas 11 (31\%) showed a significant effect of delay duration (two-way ANOVA, $p<0.05$ ).

Saccade deviation as a function of target direction and gap duration is shown in Figure $4 D$ for the 33 sites with a significant target direction effect. The single data point for a target direction of 180 and gap duration of 0 was suspiciously large. Post hoc analysis revealed that the magnitude of this deviation was attributable entirely to data from one monkey. When we excluded only the data with the gap of 0 for that monkey, the number of sites with a significant effect of gap duration fell to 5 of $33(15 \%)$. We conclude that, for the memory-guided saccade task, the evoked saccade deviation is relatively constant throughout the delay interval.

\section{Relationship between electrically evoked saccade deviation and task performance}

The fact that there was a large amount of trial-to-trial variability in the monkeys' behavior (Fig. 2) suggested that it might be worthwhile to examine the relationship between stimulation effects and task performance. Was the behavioral variability purely random, or did it reflect the degree to which monkeys were attending to the task? In the latter case, we might expect to see a relationship between performance and stimulation effects. We first looked at the effect of voluntary saccade accuracy. If inaccurate saccades reflected a failure to update the internal saccade plan, then stimulation effects should be less reliable. To test this, we divided the stimulation trials for each site into two classes: accurate and inaccurate. Accurate trials were those in which the error (eye position regarding target position) after the voluntary saccade was smaller than the median error for that site, whereas inaccurate saccades were those with errors larger than the median. This divided the data into two classes with equal number of 
Table 1. Relationship between task performance and stimulation effects for sites with a significant effect of target direction on evoked saccade deviation compared with sites with no significant effect

\begin{tabular}{|c|c|c|c|c|c|c|c|c|c|c|}
\hline \multirow{3}{*}{$\begin{array}{l}\text { Factor: target direction }{ }^{a} \\
\text { Monkey }\end{array}$} & \multicolumn{5}{|c|}{ Stimulation trials } & \multicolumn{5}{|c|}{ Nonstimulated trials } \\
\hline & \multicolumn{2}{|c|}{ Nonsignificant sites } & \multicolumn{2}{|c|}{ Significant sites } & \multirow[b]{2}{*}{$p$} & \multicolumn{2}{|c|}{ Nonsignificant sites } & \multicolumn{2}{|c|}{ Significant sites } & \multirow[b]{2}{*}{$p$} \\
\hline & $r$ & $n$ & $r$ & $n$ & & $r$ & $n$ & $r$ & $n$ & \\
\hline A & -0.346 & 39 & 0.419 & 24 & 0.002 & -0.173 & 127 & 0.189 & 90 & 0.005 \\
\hline C & 0.189 & 996 & 0.242 & 1750 & 0.07 & 0.165 & 1125 & 0.206 & 1331 & 0.15 \\
\hline$D$ & 0.241 & 2559 & 0.274 & 1028 & 0.17 & 0.244 & 2708 & 0.339 & 917 & 0.004 \\
\hline All & 0.210 & 3594 & 0.227 & 2802 & 0.22 & 0.211 & 3743 & 0.271 & 2338 & 0.006 \\
\hline
\end{tabular}

$r$, Correlation coefficient; $n$, number of saccades. The $p$ values are comparisons of the correlation coefficients for nonsignificant versus significant sites.

${ }^{a}$ Site classification and ANOVA factor.

Table 2. Relationship between task performance and stimulation effects for sites with a significant effect of gap duration on evoked saccade deviation compared with sites with no significant effect

\begin{tabular}{|c|c|c|c|c|c|c|c|c|c|c|}
\hline \multirow{3}{*}{$\begin{array}{l}\text { Factor: gap duration }{ }^{a} \\
\text { Monkey }\end{array}$} & \multicolumn{5}{|c|}{ Stimulation trials } & \multicolumn{5}{|c|}{ Nonstimulated trials } \\
\hline & \multicolumn{2}{|c|}{ Nonsignificant sites } & \multicolumn{2}{|c|}{ Significant sites } & \multirow[b]{2}{*}{$p$} & \multicolumn{2}{|c|}{ Nonsignificant sites } & \multicolumn{2}{|c|}{ Significant sites } & \multirow[b]{2}{*}{$p$} \\
\hline & $r$ & $n$ & $r$ & $n$ & & $r$ & $n$ & $r$ & $n$ & \\
\hline$A$ & -0.346 & 39 & 0.419 & 24 & 0.002 & -0.272 & 166 & 0.245 & 114 & $<0.001$ \\
\hline$C$ & 0.219 & 2099 & 0.210 & 4225 & 0.42 & 0.207 & 2228 & 0.183 & 735 & 0.28 \\
\hline$D$ & 0.239 & 1599 & 0.263 & 1988 & 0.22 & 0.246 & 1637 & 0.259 & 2042 & 0.34 \\
\hline All & 0.186 & 3737 & 0.232 & 2659 & 0.03 & 0.188 & 3775 & 0.229 & 2713 & 0.046 \\
\hline
\end{tabular}

$r$, Correlation coefficient; $n$, number of saccades. The $p$ values are comparisons of the correlation coefficients for nonsignificant versus significant sites.

${ }^{a}$ Site classification and ANOVA factor.

observations in each class. We found that when the monkey made accurate voluntary saccades, the effect of target direction on electrically evoked saccade deviation was significant at 23 of $51(45 \%)$ sites (two-way ANOVA, $p<0.05$ ). When the monkey made inaccurate saccades, the effect of target direction was significant at 13 of $51(25 \%)$ sites. The effect of delay duration was significant at $11(22 \%)$ and $10(20 \%)$ sites for accurate and inaccurate saccades, respectively (two-way ANOVA). Thus, there was more likely to be a significant deviation of the electrically evoked saccade when the monkey was performing accurately.

A complementary way to look at the relationship between task performance and stimulation effect is to ask whether performance was better for sites with significant stimulation effects than for sites with nonsignificant effects. We characterized performance in terms of the correlation between eye position after the first saccade and invisible target position, as in Figure $2 \mathrm{~A}$. The correlation coefficients are shown in Tables 1 and 2, sorted by monkey, presence of stimulation (i.e., stimulation vs nonstimulated trials), and site classification based on whether there was a significant effect of target direction on evoked saccade deviation. For sites that showed a significant effect of target direction or gap duration on electrically evoked saccade deviation, voluntary saccade performance was better in nearly every case (14 of 16 comparisons) than for sites with no significant stimulation effect. Thus, electrically evoked saccades tended to be reliably deviated when voluntary saccades were more strongly correlated with invisible target position.

Performance was further characterized in terms of whether the monkey overshot or undershot the invisible target. Overshoots were defined as trials in which the eye position eccentricity at the end of the first voluntary saccade was greater than the eccentricity of the invisible target, and undershoots were correspondingly defined as trials in which the eye eccentricity was smaller than target eccentricity. For this analysis, the total electrically evoked saccade deviation for each gap duration was calculated by taking the difference between the deviations for the two directions of target motion. The total deviation was either in the
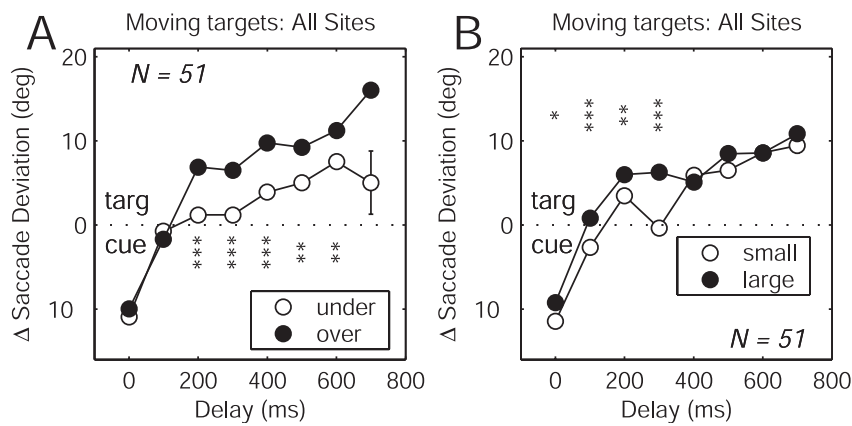

Figure 5. Dependence of evoked saccade bias on behavioral performance. $A$, Total saccade deviation as a function of delay duration for undershoot trials (open circles) and overshoot trials (filled circles). B, Saccade deviation for trials in which the voluntary saccade was smaller (open circles) or larger (filled circles) than the median. The error bars are \pm 1 SEM (most error bars were smaller than the plotted symbols). The asterisks indicate the $t$ test significance level $\left({ }^{*} p<\right.$ $\left.0.05 ;{ }^{* *} p<0.01 ;{ }^{* * *} p<0.001\right)$.

direction of the "cue" (i.e., the starting point of the target trajectory) or the "target" (i.e., the end point of the target trajectory). The change in evoked saccade deviation was significantly different for overshoots compared with undershoots (Fig. 5A). When the monkey undershot, the overall change in saccade deviation was smaller, and the deviation in the direction of the cue (early delay) was greater than the deviation in the direction of the target (later delays). When the monkey overshot, the total change in saccade deviation was greater (i.e., there was a larger difference between a delay of 0 and a delay of 600) and more in the direction of the target. The difference between overshoot and undershoot trials was more pronounced at longer delays, suggesting that the effect is related to motor planning rather than a perceptual error. These results show that on overshoot trials, the saccade plan shifted earlier and more strongly toward the end point of the target trajectory.

Another possible interpretation of the preceding result is that the evoked saccade deviation was simply correlated with the size 
of the voluntary saccades. To test this, we divided the trials into two classes based on whether the eye position eccentricity the end of the first saccade was smaller than the median eccentricity for that site (Fig. 5B, small) or larger than the median (Fig. 5B, large). Dividing the data according to saccade amplitude produced a much weaker effect compared with the effect of overshoot/undershoot. It seems that not just the size of the voluntary saccade, but also the end point of the saccade relative to target position, is important in determining the magnitude and time course of the evoked saccade deviation.

\section{Discussion}

Electrical stimulation of the prefrontal cortex elicits eye movements generally directed contralateral to the side of the stimulation site (Ferrier, 1876; Robinson and Fuchs, 1969; Bruce et al., 1985; Burman and Bruce, 1997). The prefrontal region from which eye movements are elicited by low-current electrical stimulation has been termed the FEF. Recently, a technique was developed that allows one to track the locus of spatial attention or saccade movement planning using electrical microstimulation of oculomotor command centers such as the superior colliculus or FEF (Kustov and Robinson, 1996; Gold and Shadlen, 2000, 2003). We used this technique to estimate where the monkey's locus of spatial attention or motor planning was focused when he was performing the motion extrapolation task. The present results indicate that the monkey's locus of attention or motor planning moves with the invisible target. This evidence supports the idea that monkeys perform motion extrapolation by maintaining a dynamic internal representation of the invisible moving target.

Experiments on predictive saccades to invisible targets are of interest because they offer an opportunity to study the neural basis of internal representations linking stimuli to behavioral responses. However, it is extremely difficult to actually prove that these internal representations exist in the first place. An argument can often be made that observed behavior simply results from rote learning of conditioned stimulus-response relationships. Although we took steps to reduce the likelihood that the monkeys' responses were based on such learned relationships, complex learned associations can potentially explain many behaviors without invoking intermediate representations.

In our experiments, the monkey viewed the moving target for a minimum of $500 \mathrm{msec}$. This is enough time to shift the locus of attention or a saccade plan from the initial target position to the estimated end point of the trajectory (Bisley and Goldberg, 2003). In behaviorist terms, the monkey simply had to register the stimulus position and velocity and summon the conditioned response. Given the behavioral constraints of the task (i.e., grace period duration and fixation window size), the monkeys could achieve at least $56 \%$ correct performance by making saccades with amplitudes in the range of $12.5 \pm 1.2^{\circ}$ in the direction specified by the target motion. Hence, there was little pressure for the monkeys to incorporate target motion into their saccade planning at all. One might question why the behavioral constraints were so loose. The answer is that our goal was to evoke behavior that might occur under more natural conditions. Using more stringent task constraints would certainly produce cleaner data, but only by causing a higher proportion of trials to be classified as "incorrect" and therefore excluded from additional analysis. In the present context, we feel that the notions of correct and incorrect performance are not appropriate, and this is why we sought to constrain the monkeys' behavior as little as possible. The data in Figure $2 C$ provide evidence that the task constraints did not, in fact, have a limiting effect on the monkeys' performance.
Despite the minimal task requirements, the monkeys behaved as if they were covertly tracking the invisible target as evidenced by the correlation of saccade amplitude with target eccentricity and the presence of corrective saccades in the absence of visual feedback. Furthermore, analysis of trials with multiple saccades strongly suggests that the second saccade was driven by an error signal that was updated to take into account future target location. The evidence strongly contradicted the idea that the second saccade was driven toward the same fixed location as the first saccade.

If the monkeys had used the strategy of making fixed saccades toward the target trajectory end point, then one would expect the direction of the electrically evoked saccade deviation to be relatively constant, as it was for the memory-guided saccade task. Furthermore, one would not expect the planned saccade ever to be directed toward the initial target location, because voluntary saccades to this location were almost never executed and were not rewarded.

Additional evidence for a relationship between evoked saccade deviation and task performance was provided by the following observations. First, the effect of target direction on evoked saccade deviation was more likely to be significant when the monkey made accurate voluntary saccades than when the voluntary saccade was inaccurate. Second, the magnitude of the evoked saccade deviation depended on whether the monkey overshot or undershot the target; the saccade deviation shifted farther and faster on overshoot trials. This effect was not accounted for by the magnitude of eye displacement. In general, both sets of observations suggest that deviation of the electrically evoked saccade depended on attention to and accurate performance of the task. The behavioral and stimulation results strongly suggest that monkeys naturally perform covert tracking and that a component of this tracking includes continuous updating of a saccade plan even when that involves planning saccades that are never executed.

\section{Comparison with previous studies}

Gold and Shadlen $(2000,2003)$ stimulated FEF during a motion discrimination task and found that the evoked saccade was biased in the direction of the voluntary saccade that indicated the monkey's choice. They found that the bias increased with the strength and duration of the motion stimulus. There are three main differences between their study and this one. First, in our task, there was no ambiguity about the stimulus or response, so the time course of the effect is unlikely to reflect the state of an internal decision variable as they have argued exists in their experiments. Second, the timing of their stimulation coincided with the "go" signal for the voluntary saccade so that the voluntary saccade was concatenated with the evoked saccade. Hence, their monkeys might have been in the process of initiating the voluntary saccade when the stimulation was applied. In our experiment, there was a minimum of $150 \mathrm{msec}$ between the stimulation and "go" signal, and the monkey always refixated the target in the center of the screen between the evoked and voluntary saccades. Hence, it is unlikely that our effects were attributable to "collision" of electrically evoked and voluntary saccades (Schlag and Schlag-Rey, 1990). Finally, Gold and Shadlen $(2000,2003)$ did not observe reversal of the evoked saccade deviation, as we found. In their studies, saccades were always deviated toward in the direction of the saccade target. Our finding that saccades were deviated toward the cue when stimulation was applied early during the occlusion interval may reflect the development of a motor plan that follows the imaginary target trajectory, although it is never trans- 
lated in an actual eye movement. Another interpretation is that the observed saccade modulation is attributable to an interaction of electrical stimulation with a moving attentional window. This is consistent with results of stimulation in the superior colliculus (Kustov and Robinson, 1996) and avoids stipulating a plan that is never expressed.

\section{References}

Barborica A, Ferrera VP (2002) Saccades evoked by electrical stimulation of macaque frontal eye field during covert tracking of invisible moving targets. Soc Neurosci Abstr 28:622.5.

Barborica A, Ferrera VP (2003) Estimating invisible target speed from neuronal activity in monkey frontal eye field. Nat Neurosci 6:66-74.

Bisley JW, Goldberg ME (2003) Neuronal activity in the lateral intraparietal area and spatial attention. Science 299:81-86.

Bruce CJ, Goldberg ME, Bushnell MC, Stanton GB (1985) Primate frontal eye field. II. Physiological and anatomical correlates of electrically evoked eye movments. J Neurophysiol 54:714-734.

Burman DD, Bruce CJ (1997) Suppression of task-related saccades by electrical stimulation in the primate's frontal eye field. J Neurophysiol 77:2252-2267.

Ferrier D (1876) The functions of the brain. London: Smith, Elder and Co.

Filion CM, Washburn DA, Gulledge JP (1996) Can monkeys (Macaca mulatta) represent invisible displacement? J Comp Psychol 110:386-395.

Gold JI, Shadlen MN (2000) Representation of a perceptual decision in developing oculomotor commands. Nature 404:390-394.
Gold JI, Shadlen MN (2003) The influence of behavioral context on the representation of a perceptual decision in developing oculomotor commands. J Neurosci 23:632-651.

Hooker C, Park S (2000) Trajectory estimation in schizophrenia. Schizophr Res 45:83-92.

Judge SJ, Richmond BJ, Chu FC (1980) Implantation of magnetic search coils for measurement of eye position: an improved method. Vision Res 20:535-538.

Kustov AA, Robinson DL (1996) Shared neural control of attentional shifts and eye movements. Nature 384:74-77.

Opris I, Barborica A, Ferrera VP (2001) On the gap effect for saccades evoked by electrical stimulation of the frontal eye fields in monkeys. Exp Brain Res 138:1-7.

Robinson D (1963) A method of measuring eye movement using a scleral search coil in a magnetic field. IEEE Trans Biomed Eng 10:137-145.

Robinson DA (1972) Eye movements evoked by collicular stimulation in the alert monkey. Vision Res 12:1795-1808.

Robinson DA, Fuchs AF (1969) Eye movements evoked by stimulation of frontal eye fields. J Neurophysiol 32:637-648.

Schlag J, Schlag-Rey M (1990) Colliding saccades may reveal the secret of their marching orders. Trends Neurosci 13:410-415.

Snedecor GW, Cochran WG (1989) Statistical methods. Ames, IA: Iowa State UP.

Stanton GB, Deng SY, Goldberg ME, McMullen NT (1989) Cytoarchitectural characteristic of the frontal eye fields in macaque monkeys. J Comp Neurol 282:415-427. 